\title{
TEH METABOLISM OF SUGAR PHOSPHATES IN COD MUSCLE.
}

\author{
J. R. BURT* and G. D STRoud.*
}

(Received November, 1965)

It has been known for several years that in fish muscle glycogen is metabolised through the Embden-Meyerhof pathway. Workers in many laboratories have in the course of the past fifteen years or so identified in fish muscle the majority of the intermediate compounds of this pathway and have also demonstrated the presence of most of the enzymes responsible for their interconversions. The cod musculature almost entirely consists of 'white' muscle fibres and this contains very few mitochondria." This would lead one to expect that anaerobic glycolysis (through the Embden-Meyerhof pathway) is virtually the sole energy supply for the muscle even during life.

Following our earlier work with trawled and rested cod on the levels of the various hexose phosphates ${ }^{2,3}$ in the muscle at death and during post mortem storage in ice it was felt that a similar survey of the 3-carbon components of this pathway in cod muscle would be of interest. Consequently the concentrations of some of these compounds (dihydroxyacetone phosphate, $\alpha$-glycerophosphate, glyceraldehyde 3-phosphate, 3-phosphoglyceric acid, pyruvic acid and lactic acid.) at death and during post mortem storage at $1^{\circ} \mathrm{C}$ in the muscle of rested and exercised fish were determined.

1,3-Diphosphoglyceric acid is very labile in acid conditions and if there were any present in this material it would have been hydrolysed during the extraction. However, it has been reported that in the living cell the concentration of 1,3-diphosphoglyceric acid is so low under physiological conditions that its estimation is hardly possible anyway. 2-Phosphoglyceric acid was assayed for but was found to be present in too low concentrations to be determined accurately.

Another aspect of this work which was of special interest is the phenomenon of rigor mortis. There appeared to be a number of discrepancies between the theoretical explanation for the occurrence of rigor, as had been worked out for glycerinated mammalian muscle fibres, and what had actually been observed in whole fish muscle. ${ }^{5)}$ It was felt that the redox state of the muscle might have some part to play in the development of rigor and the analytical investigations were extended to cover a number of oxidation-reduction pairs of compounds.

The compounds considered were $\mathrm{NAD}^{* *}$ and $\mathrm{NADH}_{2}, \mathrm{NADP} * *$ and $\mathrm{NADPH}_{2}$,

* Torry Research Station, Aberdeen, Ministry of Technology, Scotland.

* NAD = nicotinamide-adenine dinucleotide.

NADP $=$ nicotinamide-adenine dinucleotide phosphate. 
pyruvate and lactate, glyceraldehyde 3-phosphate and 1,3-diphosphoglyceric acid, and dihydroxyacetone phosphate and $\alpha$-glycerophosphate. The behaviour of the nucleotide coenzymes were described earlier in this symposium ${ }^{61}$, and it was found that a reliable calculation of the ratio for glyceraldehyde 3-phosphate to 1,3-diphosphoglyceric acid concentrations could not be made because of their very low values. Consequently, the remaining two pairs, pyruvate/lactate and $\alpha$-glycerophosphate/ dihydroxyacetone phosphate are the only ones for which any data are presented in this context.

The effect of exercise on rigor mortis and on the concentrations of these metabolites within cod muscle was investigated because the practical features of trawling for fish are such that an exhausted animal is produced as raw material for the processing side of the industry.

\section{Experimental}

The fish used had been maintained with feeding in our aquarium for a minimum of four weeks before they were killed. The rested fish were caught in a net and killed with as little struggling as possible by a blow on the head. A sample of muscle was removed immediately and frozen in liquid oxygen. The fish that were to be exercised were also caught in a hand net with minimum struggling. A biopsy sample was removed using a high speed dental drill fitted with a hollow needle. This gave a cylindrical shaped sample $3 \mathrm{~mm}$ in diameter, approximately $20 \mathrm{~mm}$ long and weighing 150 to $200 \mathrm{mg}$. This biopsy sample was also frozen in liquid oxygen immediately it was taken. The small hole in the fish was plugged with petroleum jelly using a syringe, length of string was tied round the fish's tail and it was then returned to the tank for exercising. This was done by tugging the string sharply, the fish would swim away and then be pulled back for stimulation again. After 20 minutes or so it would no longer respond and was assumed at this stage to be completely exhausted. The fish was then removed from the tank, killed and a death sample taken as in the case of the rested fish. All fish of both types, rested and exercised, were kept in a store room at $1^{\circ} \mathrm{C}$ until after rigor mortis had become resolved. Samples were removed from each fish at intervals throughout the storage period. Extracts of the samples were prepared and analyses carried out using modifications of the standard enzymatic methods."

\section{Results and Discussion}

Figures 1 to 5 show the concentrations of the various three-carbon metabolites in cod muscle at different times. The units of concentrations used in every case is $\mu$ Moles $/ 100 \mathrm{~g}$ muscle (wet weight). The results of the assays on the biopsy samples is shown in these figures on the extreme left of the exercised fish graphs and the changes between those concentrations and the corresponding zero-time assay shows the 
effect of exercise. The effect of exercise can also be deduced, of course, by comparing the levels of concentration in the two sets of zero-time assays. The lines joining symbols of the same type indicate that these samples were all obtained from a single fish. It will be noticed that concentrations of most of these metabolites in the biopsy samples and in the zero time samples from rested fish are very similar. The times at which the fish went into rigor have also been marked on these graphs.

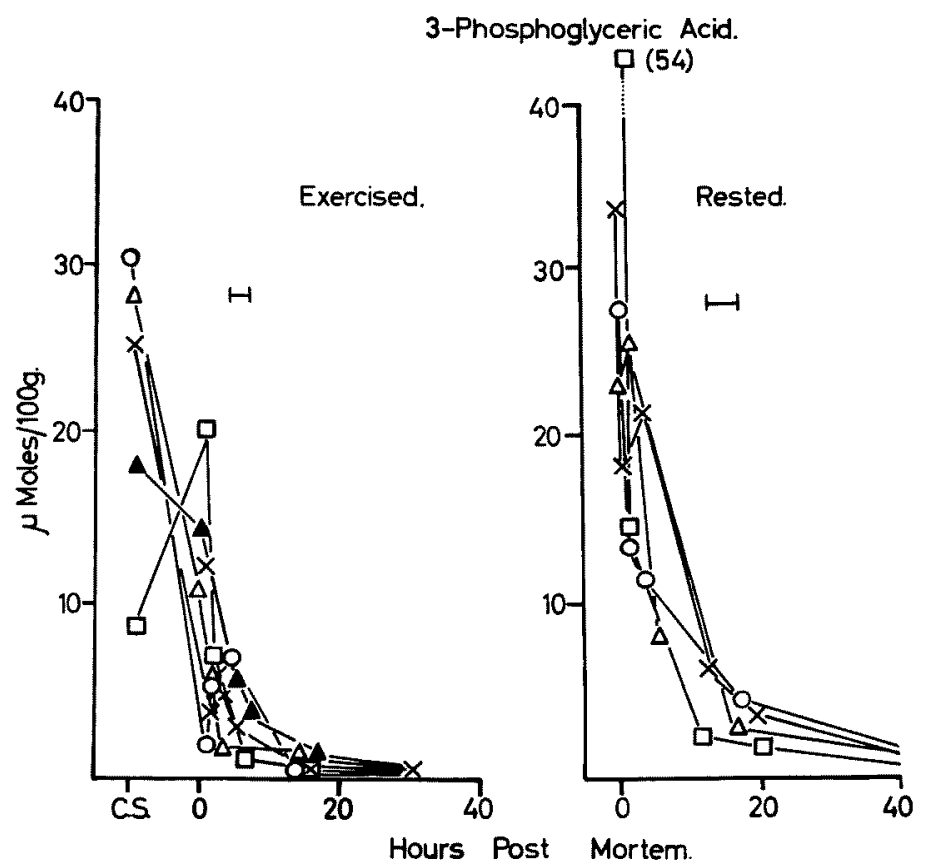

Fig. 1. Changes in the concentrations of 3-phosphoglyceric acid in cod muscle during exercise and post-mortem storage at $1^{\circ} \mathrm{C}$.

C.S.-Biopsy sample.

-Period of onset of rigor mortis.

Fructose diphosphate in normally split by aldolase to give equal amounts of dihydroxyacetone phosphate and glyceraldehyde 3-phosphate. These are interchangeable through the agency of the enzyme triose-isomerase but the equilibrium of this reaction lies so far over in favour of dihydroxyacetone phosphate that only once was any glyceraldehyde 3-phosphate detected (at a level of $0.3 \mu \mathrm{Moles} / 100 \mathrm{~g}$ at death).

3-Phosphoglyceric acid (Fig. 1) is diminished by exercise and falls rapidly to zero after death. The trends shown continue as far as 100 hours or so.

Pyruvate (Fig. 2) is present in rested cod muscle at quite low concentrations. During exercise there is an approximate threefold increase in its concentration but afrer death these levels fall to zero in a very short time. They do not change in the interval 40 to 100 hours. 
Lactate concentrations (Fig. 3) showed the usual picture of rise during exercise and also during post mortem storage. These figures show quite well the good agreement that was obtained between the biopsy samples and the zero time samples from rested fish.

Exercise produced on average a slight fall in the concentration of dihydroxyacetone phosphate (Fig. 4) but after death this fell in both types of cod to near zero

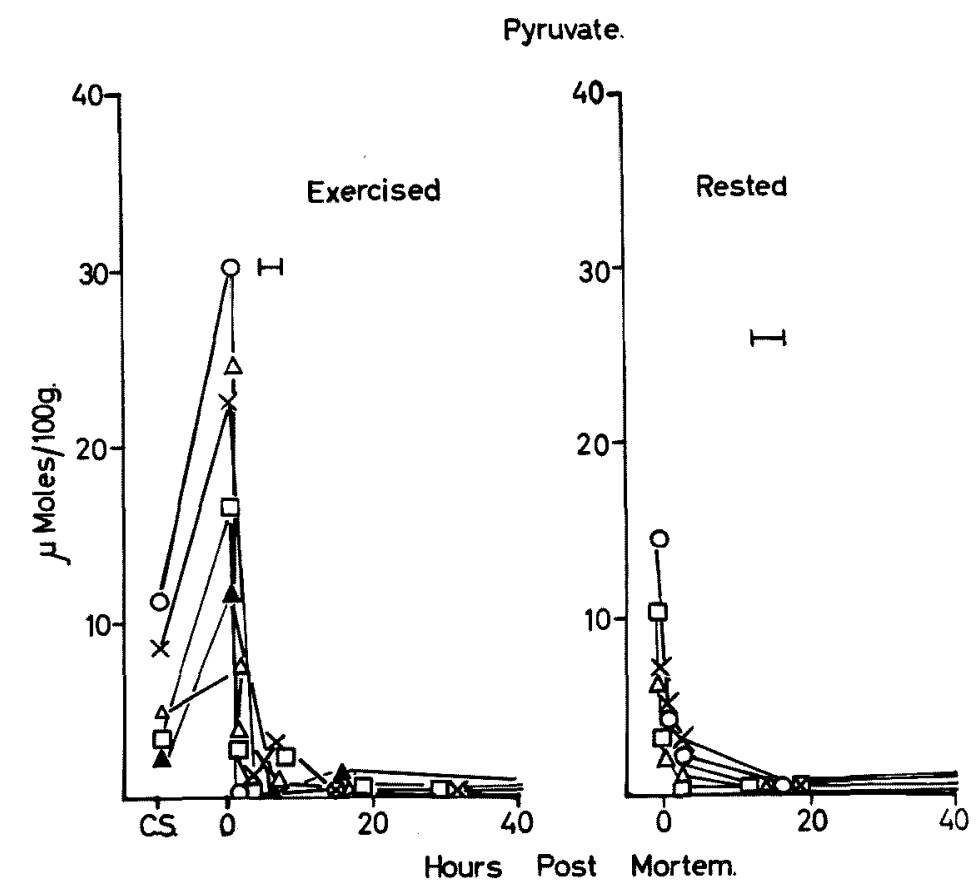

Fig. 2. Changes in the concentrations of pyruvate in cod muscle during exercise and post-mortem storage at $1^{\circ} \mathrm{C}$.

C.S.-Biopsy sample.

-Period of onset of rigor mortis.

within a day. These lines again show the actual trends that were found to occur up to 100 hours post mortem. As an alternative to the pathway through glyceral dehyde 3-phoshate to lactate, we were interested in finding out if any appreciable amount of carbohydrate would end up as $\alpha$-glycerophosphate (by the enzymic oxidation of dihydroxyacetone phosphate). $\alpha$-Glycerophosphate dehydrogenase activity has been reported in carp muscle ${ }^{8}$, but in homogenates of that tissue most of the fructose diphosphate added was metabolized to lactate and only a small proportion appeared as $\alpha$-glycerophosphate.

Cod muscle does produce a certain amount of $\alpha$-glycerophosphate during exercise and post mortem storage but very much less than it does lactate (Fig. 5). During 
storage at higher temperatures $\left(17\right.$ to $\left.20^{\circ}\right)$ it has been observed in other experiments that larger amounts of $\alpha$-glycerophosphate are produced post mortem in cod muscle.

Insect flight ${ }^{21}$ and mollusc muscles ${ }^{8)}$ are both known to be capable of producing appreciable amounts of $\alpha$-glycerophosphate and this compound has been identified as the original soluble relaxing factor in mammalian muscle. ${ }^{10}$

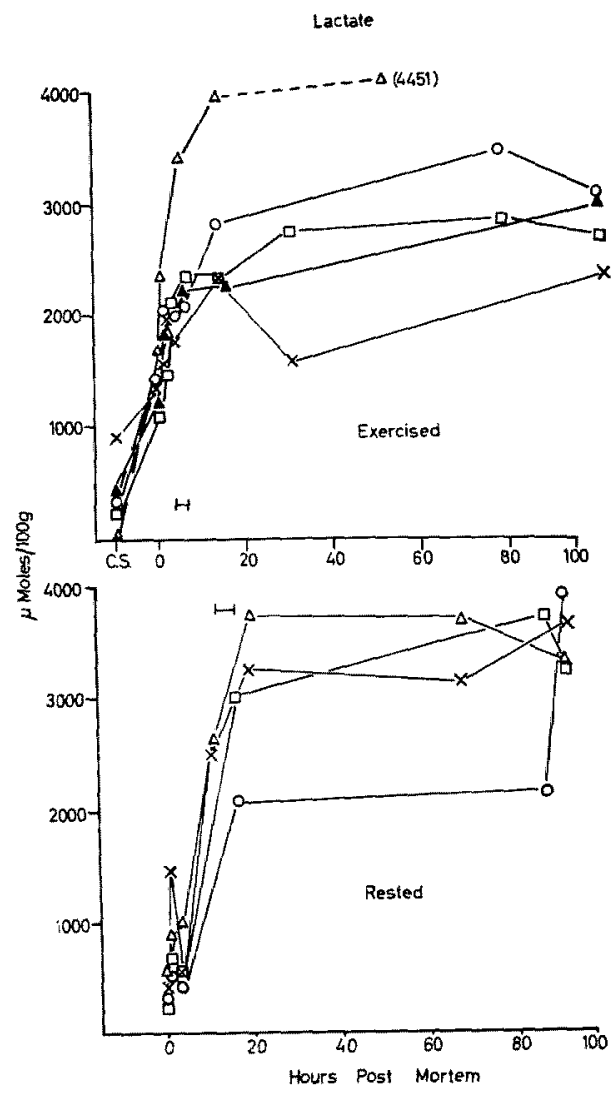

Fig. 3. Changes in the concentrations of lactate in cod muscle during exercise and post-mortem storage at $1^{\circ} \mathrm{C}$.

C.S.-Biopsy sample.

$1-1$-Period of onset of rigor mortis.

Fig. 6 shows the ratios of pyruvate to lactate and of dihydroxyacetone phosphate to $\alpha$-glycerophoshate plotted against the corresponding times post mortem. There is, in both cases, a very rapid fall in these ratios from the moment of death. It will be noticed that the ratios are not the same, there being a difference of approximately one order of magnitude between them. This presumably reflects a difference in the equilibrium constants of the two dehydrogenase enzymes. It would appear, and the data on the $\mathrm{NAD} / \mathrm{NADH}_{2}$ ratios in this same material already presented ${ }^{6 /}$ would agree 
with this, that cod muscle rapidly becomes anoxic after death.

There was not any direct relationship found between these ratios (or in fact the levels of concentration of any of the metabolites looked at) and the onset of rigor mortis. We have not so far been able to test if the redox potential of muscle and rigor mortis are related in a more complex manner-i.e. when ATP or lactate are considered-but this could be probable.

\section{Dihydroxyacetone Phosphate.}

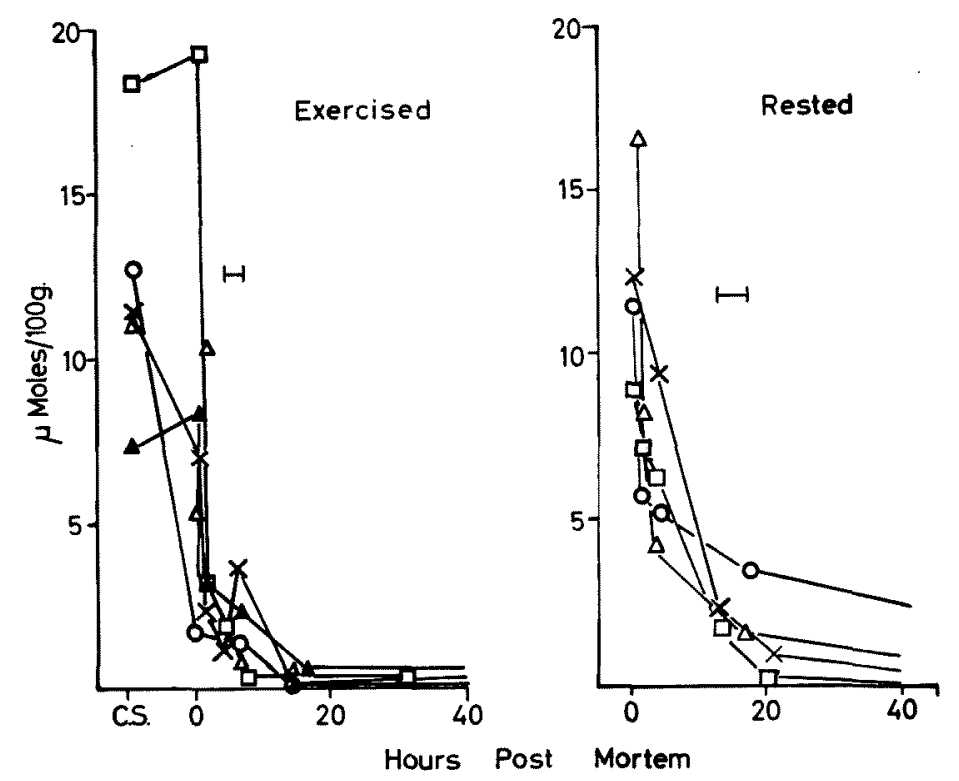

Fig. 4. Changes in the concentrations of dihydroxyacetone phosphate in cod muscle during exercise and post-mortem storage at $1^{\circ} \mathrm{C}$.

C.S.-Biopsy sample.

1 -Period of onset of rigor mortis.

There can be no doubt that the muscle of cod has the Embden-Meyerhof pathway in common with that of other animal species. As the main provider of energy by the resynthesis of ATP it is of great importance during the life of the tissue. After death the concentrations of many of these compounds can affect the properties of the fish muscle from a technological viewpoint. Many of them are flavourous. Some can react with amines in the Maillard reaction and thus produce browning of the flesh. The amount of glycogen degraded through this pathway will, by the production of lactic acid, govern the increase in acidity of the flesh and hence its keeping quality texture.

The work described in this paper was carried out as part of the programme of the Ministry of Technology. 


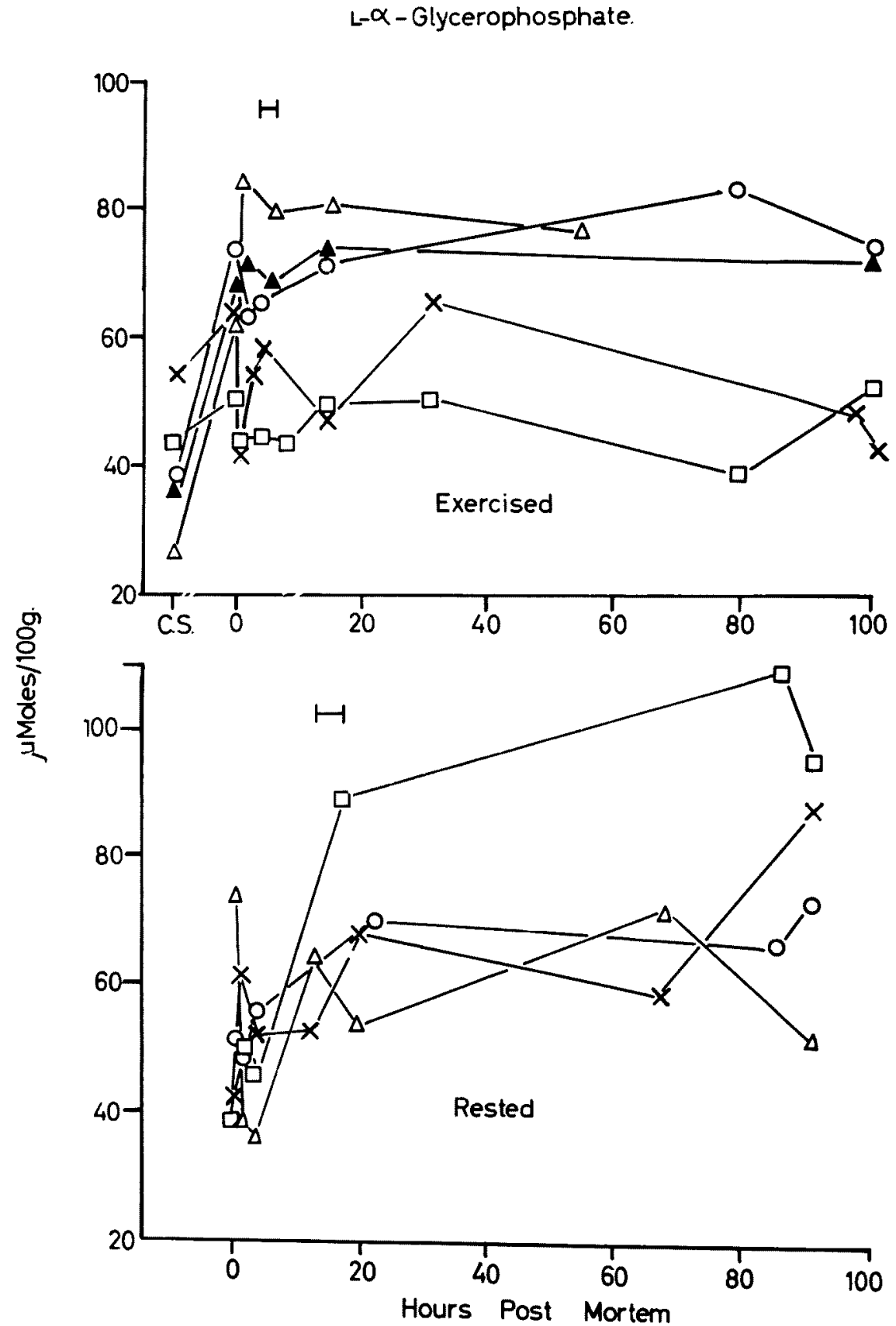

Fig. 5. Changes in the concentrations of L- $\alpha$-glycerophosphate in cod muscle during exercise and post-mortem storage at $1^{\circ} \mathrm{C}$.

C.S.-Biopsy sample.

$1-1$-Period of onset of rigor mortis. 


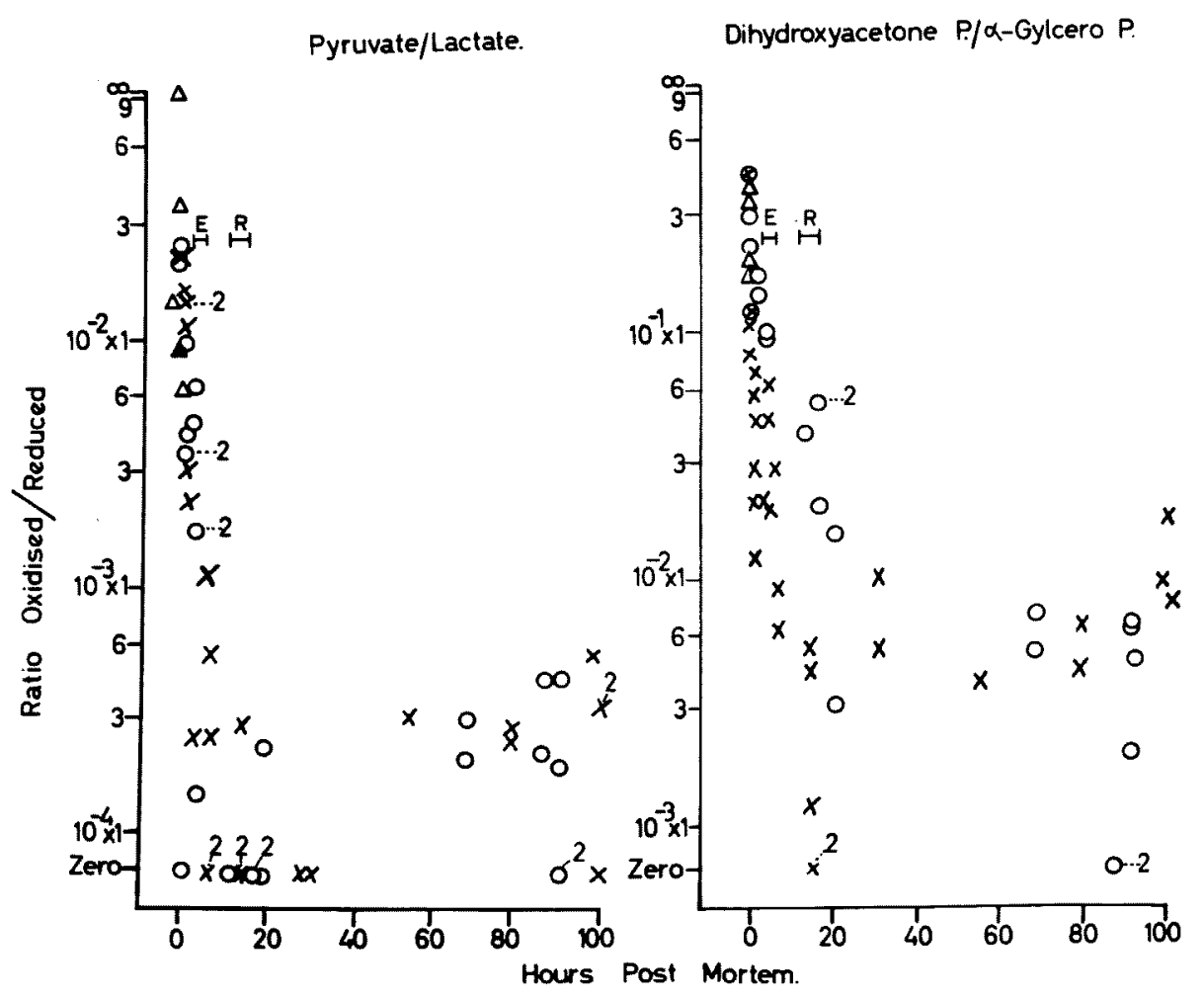

Fig. 6. Changes in the ratios of redox pairs in cod muscle during postmortem storage at $1^{\circ} \mathrm{C}$.

$x$-Exercised.

O-Rested.

Q-Rested (Biopsy).

- $1-$-Period of onset of rigor mortis for exercised and rested fish respectively. 


\section{References}

1) Howgate, P.F.: Torry Research Station. Personal Communication.

2) BURT, J.R. and Jones, N.R,: J. Sci. Fd, Agric. 12, 344 (1961).

3) Burt, J.R.: J. Food Sci. 26, 462 (1961).

4) Negelein, E.: In Methods of Enzymatic Analysis. Ed. H. U. Bergmeyer Academic Presa. 1963. p. 235.

5) Jones, N.R., Burt, J.R., Murray, J, and Stroud, G.D.: In The technology of fish utilization. Ed. R. Kreuzer, Fishing News (Books) Ltd., London. 1965 p. 14.

6) Jones, N.R. and Murray, J.: Bull. Jap. Soc. Sci. Fish. 32, 197 (1966).

7) Bergmeyer, H.U. Editor. Methods of Enzymatic Analysis. Academic Press, 1963.

8) Shibata, T. and Yoshimura, K.: Bull. Jap. Soc. Sci. Fish. 28, 514 (1962).

9) Gilmour, D.: The Metabolism of Insects. Oliver and Boyd, London. 1965 pp. 28 to 30.

10) Marsh, B. B.: Biochem. Biophys. Res. Comm. 3, 233 (1960). 